\title{
Cardiovascular risk factors associated with lower baseline cognitive performance in HIV-positive persons
}

\section{E.J. Wright, MBBS \\ B. Grund, PhD \\ K. Robertson, PhD \\ B.J. Brew, MBBS, MD \\ M. Roediger, MS \\ M.P. Bain, BSc, \\ M Clin Psych, \\ M Clin Neuropsych}

F. Drummond, MBChB, MRCA, DA (UK)

M.J. Vjecha, MD

J. Hoy, MBBS

C. Miller, MPH

A.C. Penalva de Oliveira, $\mathrm{MD}, \mathrm{PhD}$

W. Pumpradit, MD

J.C. Shlay, MD, MSPH

W. El-Sadr, MD, MPH

R.W. Price, MD

For the INSIGHT

SMART Study Group

Address correspondence and reprint requests to Dr. Edwina Wright, Infectious Diseases Unit, Alfred Hospital, Commercial Road, Melbourne, Victoria 3004, Australia

e.wright@alfred.org.au

Supplemental data at www.neurology.org

\section{ABSTRACT}

Objective: To determine factors associated with baseline neurocognitive performance in HIVinfected participants enrolled in the Strategies for Management of Antiretroviral Therapy (SMART) neurology substudy.

Methods: Participants from Australia, North America, Brazil, and Thailand were administered a 5-test neurocognitive battery. $Z$ scores and the neurocognitive performance outcome measure, the quantitative neurocognitive performance z score (QNPZ-5), were calculated using US norms. Neurocognitive impairment was defined as z scores $<-2$ in two or more cognitive domains. Associations of test scores, the QNPZ-5, and impairment with baseline factors including demographics and risk factors for HIV-associated dementia (HAD) and cardiovascular disease (CVD) were determined in multiple regression.

Results: The 292 participants had a median CD4 cell count of 536 cells $/ \mathrm{mm}^{3}, 88 \%$ had an HIV viral load $\leq 400$ copies $/ \mathrm{mL}$, and $92 \%$ were taking antiretrovirals. Demographics, HIV, and clinical factors differed between locations. The mean QNPZ-5 score was $-0.72 ; 14 \%$ of participants had neurocognitive impairment. For most tests, scores and z scores differed significantly between locations, with and without adjustment for age, sex, education, and race. Prior CVD was associated with neurocognitive impairment. Prior CVD, hypercholesterolemia, and hypertension were associated with poorer neurocognitive performance but conventional HAD risk factors and the CNS penetration effectiveness rank of antiretroviral regimens were not.

Conclusions: In this HIV-positive population with high CD4 cell counts, neurocognitive impairment was associated with prior CVD. Lower neurocognitive performance was associated with prior CVD, hypertension, and hypercholesterolemia, but not conventional HAD risk factors. The contribution of CVD and cardiovascular risk factors to the neurocognition of HIV-positive populations warrants further investigation. Neurology ${ }^{\circledR} 2010 ; 75: 864-873$

\section{GLOSSARY}

$\mathbf{A D}=$ Alzheimer disease; $\mathbf{A R T}=$ antiretroviral therapy; $\mathbf{B P}=$ blood pressure; $\mathbf{C E S}-\mathbf{D}=$ Center for Epidemiologic StudiesDepression scale; $\mathbf{C T}=$ Color Trails; CVD = cardiovascular disease; FTT = Finger Tapping Test; GPB = Grooved Pegboard; HAD = HIV-associated dementia; $\mathbf{N C I}=$ neurocognitive impairment; QNPZ-5 = quantitative neurocognitive performance $z$ score; SMART = Strategies for Management of Antiretroviral Therapy; TG = Timed Gait.

In advanced untreated HIV disease, HIV-associated dementia (HAD) develops in approximately $15 \%$ of patients ${ }^{1}$ and combination antiretroviral therapy (ART) has effectively reduced the incidence of HAD. ${ }^{2}$ The Strategies for Management of Antiretroviral Therapy (SMART) study randomized participants to intermittent, CD4-guided ART or continuous ART. ${ }^{3}$ In a neurology substudy, a neurocognitive test battery was administered. We hypothesized that neurocognitive

\footnotetext{
e-Pub ahead of print on August 11, 2010, at www.neurology.org.
}

From The Alfred Hospital (E.J.W., J.H.), Melbourne; The Burnet Institute (E.J.W.), Melbourne; Monash University (E.J.W., J.H.), Melbourne, Australia; University of Minnesota (B.G., M.R., C.M.), Minneapolis; Department of Neurology (K.R.), University of North Carolina School of Medicine, Chapel Hill; Departments of Neurology and HIV Medicine (B.J.B., M.P.B.), St Vincent's Hospital, Sydney; University of New South Wales (B.J.B.), Sydney; National Centre in HIV Epidemiology and Clinical Research (F.D.), University of New South Wales, Sydney, Australia; Washington Veterans Affairs Medical Center (M.J.V.), Washington, DC; Departamento de Neurologia (A.C.P.d.O.), Instituto de Infectologia Emílio Ribas, São Paulo, Brasil; HIV-NAT (W.P.), Bangkok, Thailand; Denver Public Health (J.C.S.), Denver, CO; International Center for AIDS Care and Treatment Programs (W.E.-S.), Mailman School of Public Health, Columbia University, New York, NY; and Department of Neurology (R.W.P.), University of California, San Francisco.

Study funding: Supported by the NIH (NIMH/NINDS U01-AI4636, NIAID U01AI042170, and NIAID U01AI46362).

Disclosure: Author disclosures are provided at the end of the article. 
performance would be superior in patients receiving continuous ART via its attendant benefits upon both peripheral and CNS immunity.

We present a cross-sectional analysis of 292 HIV-infected persons coenrolled in the SMART neurology substudy at sites in Australia, North America, Brazil, and Thailand. We sought to explore factors associated with neurocognitive performance. These included demographics, ART, HAD, and cardiovascular risk factors and cardiovascular disease (CVD). In HIV-negative populations, smoking, ${ }^{4}$ hypertension, ${ }^{5}$ high cholesterol, ${ }^{6}$ obesity, ${ }^{5}$ and diabetes ${ }^{5}$ are the cardiovascular risk factors associated with increased risk of poor cognitive function, vascular dementia, and Alzheimer disease (AD). Prior myocardial infarction, ${ }^{7}$ coronary artery bypass grafting, ${ }^{8}$ and stroke ${ }^{5}$ are also associated with poor cognitive function. In HIV-positive populations, diabetes has been associated with HAD, ${ }^{9}$ and increased carotid intima media thickening ${ }^{10}$ has been associated with poorer neurocognitive performance. CVD risk factors were common in the SMART study ${ }^{3}$ : we hypothesized that they would be associated with lower baseline neurocognitive performance. We sought to describe and compare neuropsychological test results obtained in Australia, North America, Brazil, and Thailand and hypothesized that test scores, but not the standardized $z$ scores, would differ across locations.

METHODS Study design. The SMART study was an international randomized trial comparing continuous ART with CD4-cell count guided, intermittent ART in HIV-infected persons with CD4 cell count $>350$ cells $/ \mathrm{mm}^{3}$. The neurocognitive component of the SMART neurology substudy aimed to compare the 2 study arms for changes in neurocognitive function through follow-up. Baseline, month 6 , and annual assessment of neurocognitive functioning in 600 participants would have provided $80 \%$ power to detect a difference of 0.27 in the primary outcome (change in the aggregate quantitative neurocognitive performance $z$ score, QNPZ-5, described below). The neurology substudy commenced enrollment in July 2005. In January 2006, enrollment and the intermittent CD4-guided ART strategy of the SMART study were stopped due to increased risk of AIDS and serious non-AIDS complications. The primary results of the SMART study are published elsewhere. ${ }^{3}$ At the time of this protocol change, only 292 of the 600 planned substudy participants were enrolled, and minimal follow-up data were available.

Forty-seven SMART study sites in Australia, North America, Brazil, and Thailand participated in the substudy. All eligible SMART participants were offered substudy coenrollment. Substudy eligibility criteria included age $\geq 18$ years, and the abil- ity to perform the study's neurocognitive tests in the site clinician's judgment.

Standard protocol approvals, registrations, and patient consents. Substudy approval was obtained by each site's institutional review board. Patient information and consent forms were translated into Thai, Portuguese, and Spanish, and backtranslated into English. All participants provided written informed consent. The study was registered at clinical trials.gov: NCT00432003.

Outcome measures. The study's neurocognitive test battery comprised the Grooved Pegboard (GPB) (dominant hand), ${ }^{11}$ Color Trails (CT) 1 and CT2 ${ }^{12}$ Timed Gait (TG), ${ }^{13}$ and Finger Tapping Test (FTT) (nondominant hand). ${ }^{14}$ Details of these tests can be found in appendix e-1 on the Neurology ${ }^{\circledR}$ Web site at www.neurology.org. This test battery was chosen because it is sensitive to changes observed in HIV-associated neurocognitive disorders, brief, easy to administer and teach, and can be used internationally where little formal Western education exists ${ }^{15}$; our tests closely resemble the battery used in a large, randomized ART HIV treatment study. ${ }^{16}$

For all 5 tests, the raw scores of each participant were standardized to $z$ scores by subtracting the mean test scores of matched, HIV-negative reference populations, and dividing by the reference SD. Therefore, $z$ scores estimate how many SDs a test score is above or below the average score of the reference population; negative $z$ scores denote below-average performance. For the TG, raw scores were standardized to $z$ scores using previously published reference distributions, ${ }^{13}$ matched by education level. For the other tests, $z$ scores were calculated using scoring software by Psychological Assessment Resources, Inc., Odessa, FL. ${ }^{17}$ Reference distributions were matched by education level for all 5 tests, and additionally by age, sex, and race/ethnicity for GPB and FTT, ${ }^{18}$ and by age for CT. All reference populations were obtained in the United States.

Our primary neurocognitive performance outcome measure was the QNPZ-5, calculated as the average of the $5 z$ scores from the individual tests in the battery. A QNPZ-5 score below 0 denotes below-average neurocognitive performance. For the purpose of this study, we defined neurocognitive impairment (NCI) as $z$ scores $<-2$ in at least 2 cognitive ability domains. Ability domains assessed were 1) speed/fine motor skills (GPB and FTT), 2) attention/ speed of processing (CT1), 3) abstraction/executive function (CT2), and 4) gross motor skills (TG). The cutoff was chosen to reflect criteria for abnormal neurocognitive performance that are a required component for the diagnosis of HAD. ${ }^{19}$

We administered the Center for Epidemiologic Studies-Depression scale $(\mathrm{CES}-\mathrm{D})^{20}$ to screen for depression at baseline. The CES-D has been used in international studies in HIV-infected ${ }^{15}$ populations. We used the recommended cutoff score of $\geq 16$ to define depression (sensitivity $86 \%-100 \%$, specificity $53 \%-84 \%$ ). ${ }^{21}$

Neuropsychological tests and the CES-D were administered to participants by site staff, including medical practitioners, specialists, and nurses. Site staff underwent centralized training and certification in the United States and Australia. Training was led by a neuropsychologist or neurologist and an infectious disease physician. Each trainee was required to administer the full test battery during training, and at least 3 times afterwards to nonstudy participants before administering it to study participants. Test instructions and training materials were available for site staff on the study Web site. Neuropsychological test instructions were translated into Portuguese, Spanish, and Thai. We used published translations of the Spanish ${ }^{22}$ and Brazilian Portuguese ${ }^{23} \mathrm{CES}-\mathrm{D}$ and an online Thai ${ }^{24}$ translation. 
Table 1 Baseline characteristics by location

\begin{tabular}{|c|c|c|c|c|c|}
\hline \multirow[b]{2}{*}{ Characteristics } & \multirow[b]{2}{*}{$\begin{array}{l}\text { Total }(n=292) \\
\% \text { or median (IQR) }\end{array}$} & \multicolumn{3}{|c|}{ Location, \% or median } & \multirow[b]{2}{*}{$\begin{array}{l}\text { p Value } \\
\text { (location) }\end{array}$} \\
\hline & & $\begin{array}{l}\text { Australia and North } \\
\text { America }(n=102)\end{array}$ & $\begin{array}{l}\text { Brazil } \\
(n=45)\end{array}$ & $\begin{array}{l}\text { Thailand } \\
(n=145)\end{array}$ & \\
\hline \multicolumn{6}{|l|}{ Demographics } \\
\hline Age, $y$ & $40(35-45.5)$ & 43 & 42 & 38 & $<0.001^{a}$ \\
\hline Female & 41.8 & 18.6 & 37.8 & 59.3 & $<0.001^{a}$ \\
\hline Race/ethnicity & & & & & $<0.001^{a}$ \\
\hline Asian & 50.7 & 2.0 & 2.2 & 100.0 & \\
\hline Black & 19.5 & 45.1 & 24.5 & 0 & \\
\hline Other or unknown & 29.8 & 52.9 & 73.3 & 0 & \\
\hline Education (\% with $\leq 12$ y) & 53.8 & 40.2 & 75.6 & 56.6 & $<0.001^{a}$ \\
\hline \multicolumn{6}{|l|}{ HIV-related factors } \\
\hline CD4 count, cells $/ \mathrm{mm}^{3}$ & $536(437-693)$ & 522 & 628 & 517 & $0.003^{a}$ \\
\hline Nadir CD4 count, cells $/ \mathrm{mm}^{3}$ & $225(158-296)$ & 208 & 234 & 226 & 0.99 \\
\hline HIV RNA $\leq 400$ copies $/ \mathrm{mL}$ & 87.6 & 69.6 & 88.6 & 100.0 & $<0.001^{\mathrm{a}}$ \\
\hline Prior AIDS diagnosis & 20.9 & 26.5 & 44.4 & 9.7 & $<0.001^{\mathrm{a}}$ \\
\hline \multicolumn{6}{|l|}{ Preexisting conditions } \\
\hline$C V D^{b}$ & 3.4 & 5.9 & 4.4 & 1.4 & 0.15 \\
\hline Diabetes & 3.8 & 4.9 & 4.4 & 2.8 & 0.66 \\
\hline \multicolumn{6}{|l|}{ ART use at baseline } \\
\hline Years of prior ART use & $4(3-6)$ & 5 & 6 & 3 & $<0.001^{a}$ \\
\hline Current ART use & 92.5 & 82.4 & 97.8 & 97.9 & $<0.001^{a}$ \\
\hline $\begin{array}{l}\text { CNS penetration } \\
\text { effectiveness rank }^{c}\end{array}$ & $1.5(1.5-2.0)$ & 1.5 & 2.0 & 1.5 & $<0.001^{\mathrm{a}}$ \\
\hline \multicolumn{6}{|l|}{ Cardiovascular risk factors } \\
\hline Total cholesterol, mg/dL & $196(168-222)$ & 190 & 180 & 203 & $0.02^{\mathrm{a}}$ \\
\hline HDL cholesterol, mg/dL & $44(36-54)$ & 41 & 37 & 49 & $<0.001^{a}$ \\
\hline Lipid-lowering drugs & 8.6 & 18.6 & 6.7 & 2.1 & $<0.001^{\mathrm{a}}$ \\
\hline $\begin{array}{l}\text { Blood pressure-lowering } \\
\text { drugs }\end{array}$ & 11.0 & 21.6 & 13.3 & 2.8 & $<0.001^{a}$ \\
\hline Body mass index, $\mathrm{kg} / \mathrm{m}^{2}$ & $23.5(21.1-26.6)$ & 25.1 & 24.2 & 22.2 & $<0.001^{a}$ \\
\hline Current smoking & 23.3 & 44.1 & 13.3 & 11.7 & $<0.001^{\mathrm{a}}$ \\
\hline Recreational drug use & 5.5 & 13.7 & 2.2 & 0.7 & $<0.001^{\mathrm{a}}$ \\
\hline $\begin{array}{l}\text { Predicted } 10-y \text { risk of } C V D^{d} \\
\text { (\% per } 10 y)\end{array}$ & $3(2-7)$ & 5 & 4 & 3 & $<0.001^{a}$ \\
\hline
\end{tabular}

Abbreviations: ART = antiretroviral therapy; CVD = cardiovascular disease; HDL = high-density lipoprotein; IQR $=$ interquartile range.

a Significant.

b Previous myocardial infarction (4), stroke (0), coronary heart disease (3), congestive heart failure (2), or peripheral vascular disease (5).

${ }^{c}$ Calculated using the CNS Penetration Effectiveness Rank. ${ }^{26}$

d Calculated using the Framingham score. ${ }^{25}$

Baseline data collected within the parent SMART study included demography, HIV history, general medical history, and laboratory values (summarized in table 1 and table e-1); additionally, alcohol and drug use were collected within the substudy. We summarized risk factors for CVD into a modified Framingham score; since blood pressure (BP) was not available, we assigned a systolic $\mathrm{BP}$ of $140 \mathrm{~mm} \mathrm{Hg}$ to participants using antihypertensive drugs, and $120 \mathrm{~mm} \mathrm{Hg}$ otherwise. ${ }^{25}$ We calculated the CNS penetration effectiveness rank ${ }^{26}$ of patients' ART regimens.

Statistical methods. Baseline characteristics, neuropsychological test scores, and CES-D scores were summarized by location of enrollment. The Kruskal-Wallis rank sum test was used to compare median values across locations, $\chi^{2}$ tests to compare percentages. Mean test scores (raw scores and $z$ scores) were compared across locations using analysis of variance and the Tukey Honest Significant Difference pairwise comparison method. In order to account for variability between clinical sites within countries, we also compared locations in hierarchical mixed effects models with sites nested in location.

Associations of baseline factors with raw test scores, $z$ scores, the QNPZ-5 score, and having $z$ scores $<-2$ in 2 or more cognitive domains (a total of 12 outcomes) were determined by multiple linear or logistic regression. In a first step, the following factors were included: HAD risk factors (age, current and nadir 
CD4 cell counts, prior AIDS, diabetes); sex; race/ethnicity; location of enrollment; education; body mass index; smoking; alcohol abuse; recreational drug use; history of hepatitis $\mathrm{B}$, hepatitis C, or CVD; viral load (HIV RNA $\leq 400$ copies/mL); CNS penetration effectiveness rank; use of BP-lowering and lipidlowering drugs; total cholesterol, high-density lipoprotein, and low-density lipoprotein; and CES-D score $\geq 16$. Factors were then eliminated using backwards selection with the Akaike information criterion. We retained age, gender, race, and education in all models, because these factors were associated with neuro- psychological test results in noninfected populations, and reference norms for calculating $z$ scores are usually matched by these factors. We also retained location to consistently assess the location effect. Coefficients and $p$ values from the final models were presented. In a separate model, lipids and use of BP-lowering drugs were replaced by Framingham risk estimates for CVD (4 categories), ${ }^{25}$ extrapolating BP from use of BP-lowering drugs.

Analyses were performed using SAS version 9.2 (SAS Institute, Cary, NC) and $R$ version 2.9. All tests were 2-sided. $p$ Values $\leq 0.05$ were considered significant.

Table 2 Neuropsychological test performance and CES-D scores by location ${ }^{a}$

\begin{tabular}{|c|c|c|c|c|c|c|c|c|}
\hline & \multirow[b]{2}{*}{$\begin{array}{l}\text { Total } \\
(n=292)\end{array}$} & \multicolumn{4}{|l|}{ Location } & \multicolumn{3}{|c|}{ Hierarchical model ${ }^{c}$} \\
\hline & & $\begin{array}{l}\text { Australia and } \\
\text { North America } \\
(n=102)\end{array}$ & $\begin{array}{l}\text { Brazil } \\
(n=45)\end{array}$ & $\begin{array}{l}\text { Thailand } \\
(n=145)\end{array}$ & $\begin{array}{l}\text { p Value, } \\
\text { location } \\
\text { ( } n=292)\end{array}$ & $\begin{array}{l}\text { SD, } \\
\text { between } \\
\text { sites }\end{array}$ & $\begin{array}{l}\text { SD, } \\
\text { between } \\
\text { patients }\end{array}$ & $\begin{array}{l}p \text { Value, }{ }^{c} \\
\text { location } \\
(n=272)\end{array}$ \\
\hline \multicolumn{9}{|l|}{$\begin{array}{l}\text { Test scores, } \\
\text { mean } \pm S D\end{array}$} \\
\hline CT1, s & $49.5 \pm 22.5$ & 48.8 & 60.2 & 46.7 & $0.002^{d}$ & 7.9 & 21.5 & 0.27 \\
\hline CT2, s & $98.6 \pm 43.0$ & 92.3 & 127.1 & 94.1 & $<0.001^{d}$ & 15.8 & 40.2 & 0.08 \\
\hline TG, s & $11.9 \pm 2.1$ & 11.8 & 11.2 & 12.1 & $0.04^{d}$ & 0.8 & 2.0 & 0.51 \\
\hline GPB & $-0.26 \pm 1.19$ & -0.62 & -0.04 & -0.08 & $<0.001^{d}$ & 0.22 & 1.10 & $0.04^{d}$ \\
\hline СТ1 & $-0.63 \pm 1.42$ & -0.63 & -1.16 & -0.47 & $0.02^{d}$ & 0.53 & 1.35 & 0.50 \\
\hline СТ2 & $-0.15 \pm 1.27$ & -0.06 & -0.85 & 0.00 & $<0.001^{d}$ & 0.50 & 1.19 & 0.20 \\
\hline TG & $-1.60 \pm 1.74$ & -1.60 & -0.94 & -1.82 & $0.01^{d}$ & 0.58 & 1.62 & 0.29 \\
\hline FTT & $-0.96 \pm 1.10$ & -1.09 & -0.41 & -1.04 & $0.001^{d}$ & 0.45 & 1.04 & 0.25 \\
\hline QNPZ-5, mean \pm SD & $-0.72 \pm 0.84$ & -0.80 & -0.68 & -0.68 & 0.53 & 0.35 & 0.79 & 0.81 \\
\hline $\begin{array}{l}\text { No. }(\%) \text { with } z \\
\text { scores <-1 in } 2 \text { or } \\
\text { more independent } \\
\text { domains }\end{array}$ & $150(51.4)$ & 52 (51.0) & $18(40.0)$ & 80 (55.2) & 0.21 & & & \\
\hline $\begin{array}{l}\text { Depression: } \\
\text { No. }(\%) \text { with } \\
\text { CES-D } \geq 16\end{array}$ & $71(25.5)$ & 38 (41.3) & 14 (31.1) & 19 (13.5) & $<0.001^{d}$ & & & \\
\hline
\end{tabular}

Abbreviations: CES-D = Center for Epidemiologic Studies-Depression scale; CT = Color Trails; FTT = Finger Tapping Test (nondominant hand); GPB = Grooved Pegboard (dominant hand); $\mathrm{NCl}=$ neurocognitive impairment; QNPZ-5 = quantitative neurocognitive performance $z$ score; $\mathrm{TG}=$ Timed Gait.

a Test scoring: GPB: time in seconds. CT1 and 2 tests: time in seconds. TG: 3 timed trials; scored as average time per trial, in seconds. FTT: each participant performed between 5 and 10 trials. The test score was the average of the 5 trials where the number of taps had the least spread. Outliers: We capped the time recorded for the GPB test at 300 seconds and for TG at 45 seconds (1 participant each). Test scores that corresponded to $z$ scores that were more than 11 SD above or below the reference mean were considered recording errors and deleted from the analysis (1 CT1, and 2 TG records).

${ }^{b}$ Differences between locations were tested in an analysis of variance model.

c Differences between locations were tested in a mixed effects hierarchical model, with random effects for sites, nested in location, and fixed effects for the 3 locations. This analysis includes 272 participants at 15 sites (Australia 14/1, North America 68/7, Brazil 45/2, Thailand 145/5); clinical sites with less than 5 participants were pooled by city, or by country, or deleted.

d Significant.

e $z$ Scores were standardized by subtracting the mean of reference distributions (test scores of healthy individuals matched for education [all tests], age [GPB, CT, FTT], gender [GPB, FTT], race/ethnicity [GPB, FTT]), and dividing by the reference distribution's standard deviation. In all locations, mean $z$ scores were below $0(p<0.001)$; $z$ scores below 0 denote below-average performance. 
RESULTS Baseline characteristics. Baseline characteristics of the 292 study participants by their location of enrollment are shown in table 1 and table e-1. Participant characteristics differed across locations, including their demographics, HIV, and general medical history.

Baseline neurocognitive test scores are summarized by location in table 2 and the figure. Overall, the mean QNPZ-5 score was -0.72; in each location, the mean $z$ score was below $0(p<0.001)$, denoting below-average performance compared to a healthy, matched population. Fourteen percent of patients met the study definition of NCI $(z$ scores $<-2$ in 2 or more cognitive domains); $51 \%$ of participants had $z$ scores $<-1$ in 2 or more cognitive domains. For each of the 5 tests, mean $z$ scores differed between locations (all $p \leq 0.02$ ). There was no evidence, however, for regional differences in mean QNPZ-5 scores $(p=0.53)$, and no one region performed consistently higher or lower across all tests. In pairwise comparisons, there were no significant differences in test scores between Australia/North America and Thailand, except for the GPB (figure). Table 2 also shows the variability in test scores between clinical sites (between-site SD) in relation to the between-patient $\mathrm{SD}$, estimated in hierarchical mixed models. For all tests except GPB, the between-site SD was about one-third to one-half of the between-patient SD, and remaining differences between locations (countries) were not statistically significant relative to the differences between sites $(p>0.05$ in the hierarchical models).

The mean CES-D score was 11.2. Overall, 71 participants (26\%) met the study definition of depression (table 2). The proportion of participants with depression was highest in Australia/North America (41\%) and lowest in Thailand (14\%).

Association between baseline factors and neurocognitive performance. The $z$ scores for all 5 tests, but not

Figure $\quad z$ Scores and summary QNPZ-5 for 5 neuropsychological tests, by location

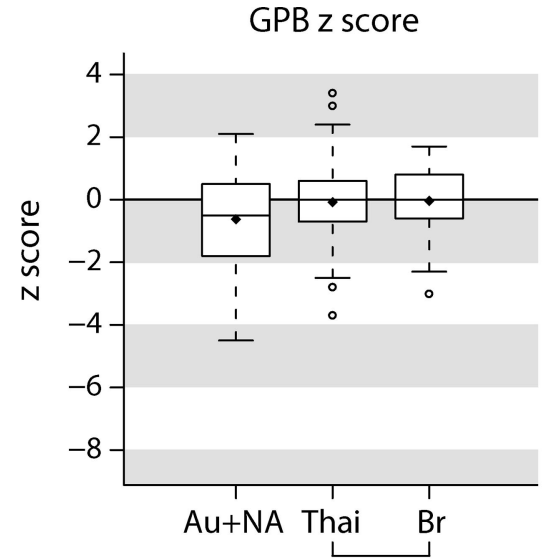

TG z score

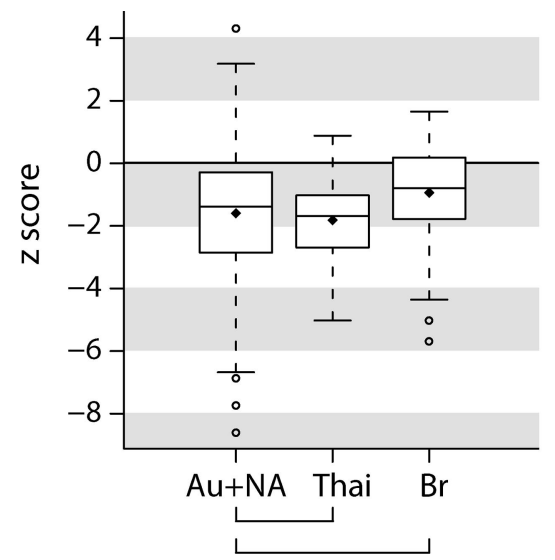

CT1 z score

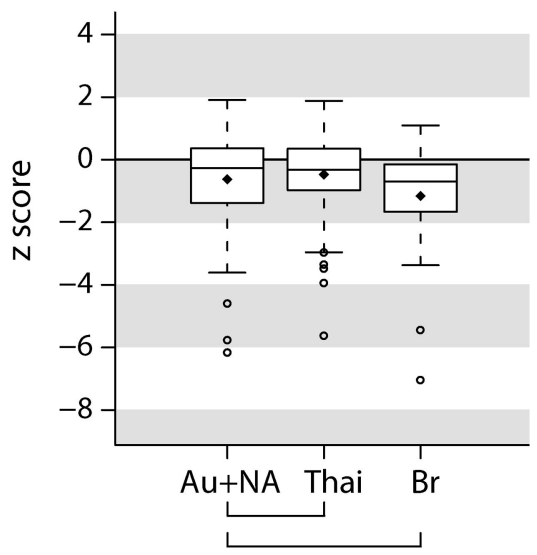

FT z score

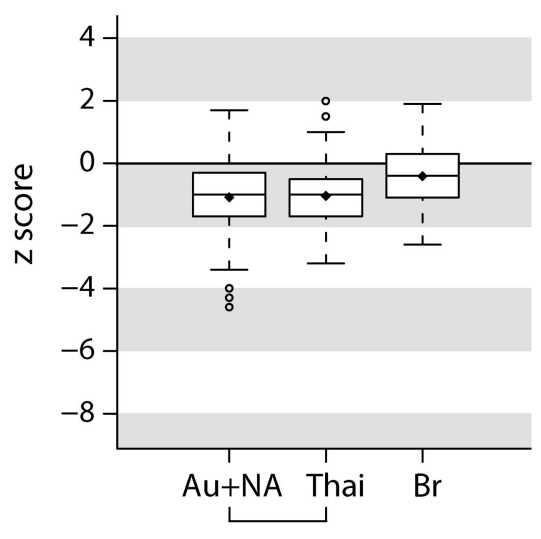

CT2 z score

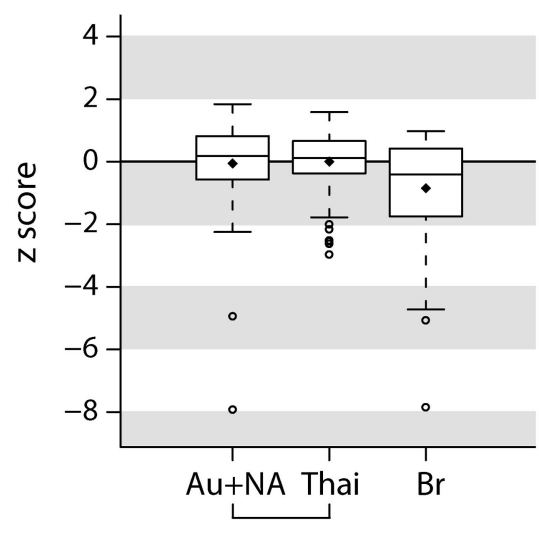

QNPZ-5 score

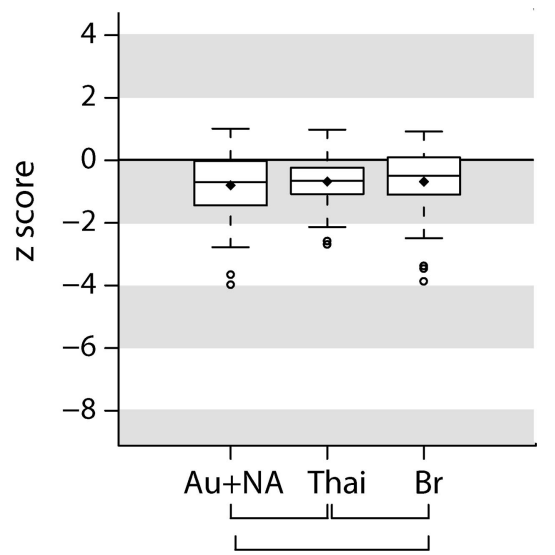

Box plots show the distributions of baseline z scores of 292 participants; 102 participants were enrolled in Australia and North America, 45 in Brazil, and 145 in Thailand. Brackets below the location labels connect pairs of locations where mean scores are not significantly different. Boxes show the interquartile range; the horizonta line in the box denotes the median; the diamond denotes the mean. Whiskers extend 1.5 times the interquartile range above and below the $z$ score quartiles. Circles denote outliers. Au = Australia; $\mathrm{Br}=$ Brazil; CT = Color Trails Test; FT = Finger Tapping Test; GPB = Grooved Pegboard Test; NA = North America; QNPZ-5 = quantitative neurocognitive performance $z$ score; TG = Timed Gait Test; Thai = Thailand. 
the QNPZ-5 scores, differed by location after adjustment for age, gender, race/ethnicity, education, and selected other factors (table 3). Older age was associated with worse test performance in raw test scores except for TG, but not with any of the $z$ scores, which are standardized by age. Women and black participants had lower estimated mean QNPZ-5 scores (by 0.21 and 0.48 ) after adjustment for the other covariates.

Patients with preexisting CVD had 6.2-fold higher odds of having NCI ( $p=0.01,95 \%$ CI $1.4-$ 26.4), after adjustment for age, gender, race/ethnicity, education, location, prior AIDS, and total cholesterol (table 3). Prior CVD was also associated with lower QNPZ-5 scores (by $-0.7 ; p=0.02$ ), as were use of antihypertensive agents (by $-0.4, p=$ 0.03 ), higher total cholesterol (by -0.03 per $10 \mathrm{mg} /$ $\mathrm{mL}, p=0.02$ ), and hepatitis B (by $-0.7, p=0.05$ ); estimated mean QNPZ-5 scores were lower for women, black participants, and, borderline, for those with depression ( $p=0.07)$ (table 3). Smoking, diabetes, higher body mass index, higher low-density lipoprotein, and use of lipid-lowering drugs were not independently associated with NCI or lower QNPZ-5 scores but were associated with lower $z$ scores or worse test scores for some of the tests (table 3 and table e-2). Higher high-density lipoprotein was associated with lower $z$ scores on GPB and CT1, but not with NCI or QNPZ-5 scores. In a sensitivity analysis, we included the Framingham CVD risk score as a factor instead of fitting cholesterol and BPlowering drugs separately: there was no evidence for an association of the Framingham score with either the QNPZ-5 or NCI (data not shown). There was no evidence that major abnormalities on the baseline ECG, hepatitis C, or alcohol abuse were independently associated with any of the neurocognitive performance measures (table 3, footnote).

There was no evidence for an association of baseline or nadir CD4 cell counts, viral load, diabetes, or the CNS penetration effectiveness rank of ART regimens with QNPZ-5 scores or NCI.

DISCUSSION Our cross-sectional study of baseline neurocognitive performance included 292 SMART study participants from Australia, North America, Brazil, and Thailand with CD4 cell counts $>350$ cells $/ \mathrm{mm}^{3}$. At baseline, $14 \%$ of participants had NCI and prior CVD was the single associated factor. Participants with prior CVD, higher total cholesterol, and those using antihypertensive drugs had lower estimated mean baseline neurocognitive performance, but neither HIV-related risk factors commonly associated with $\mathrm{HAD}$ nor the CNS penetration effectiveness rank of ART were associated with $\mathrm{NCI}$ or neurocognitive performance measured by the QNPZ-5. The 10 participants (3.4\%) with prior CVD in our study had experienced myocardial infarction, coronary heart disease, congestive heart failure, or peripheral vascular disease, but not stroke.

Our findings suggest that cardiovascular risk factors and disease may be key drivers of impairment in HIV-positive persons with high CD4 cell counts, more so than HIV-related HAD risk factors. These findings are important because HIVpositive populations have a high number of CVD risk factors including smoking, ${ }^{3}$ hypertension, ${ }^{3} \mathrm{di}-$ abetes, ${ }^{3}$ hyperlipidemia, ${ }^{3}$ metabolic syndrome, ${ }^{27}$ insulin resistance, ${ }^{28}$ and antiretroviral use. ${ }^{29}$

The mechanisms that might link lower neurocognitive performance with CVD and cardiovascular risk factors in HIV-positive populations are likely multifactorial. In HIV-negative populations increasing evidence suggests that cardiovascular risk factors contribute to the pathogenesis of both vascular dementia and AD. ${ }^{4-6,30}$ Theoretically the pathogenesis of these 2 dementing illnesses may overlap. Recently it was proposed that insulin resistance, which underlies several of the abovementioned cardiovascular risk factors, may be one of the primary convergent mechanisms that links these risk factors to both vascular dementia and $\mathrm{AD} .{ }^{31}$

Raised inflammatory markers, including IL- 6 and C-reactive protein, are risk factors for dementia ${ }^{32}$ and are common to $\mathrm{CVD},{ }^{33}$ the metabolic syndrome, ${ }^{34}$ aging, and HIV, including treated HIV infection. ${ }^{35}$ However, we do not have inflammatory markers for study analysis.

The changes in the adaptive immune system of HIV-infected patients receiving long-term antiretroviral therapy resemble those seen in the elderly. ${ }^{36} \mathrm{Key}$ factors that may contribute to immune senescence in HIV-infected patients include persistent immune activation, inflammation, and poor $\mathrm{CD} 4$ cell response to antiretroviral treatment. ${ }^{36} \mathrm{~A}$ recent study of HIVinfected patients measured coronary artery calcium accumulation and found an increased vascular age in over $40 \%$ of patients, with a mean increase of 15 years over their chronological age. ${ }^{37}$ Increased vascular aging would help to explain why we found an association between lower neurocognitive performance and cardiovascular risk factors/CVD in a relatively young study population whereas cognitive decline and dementia associated with these factors in HIV-negative populations usually do not occur until at least the sixth decade. Similarly, the early expression of Parkinson disease in HIV infection has been reported recently. ${ }^{38}$

None of the conventional HIV-related HAD risk factors was associated with NCI or lower neurocogni- 


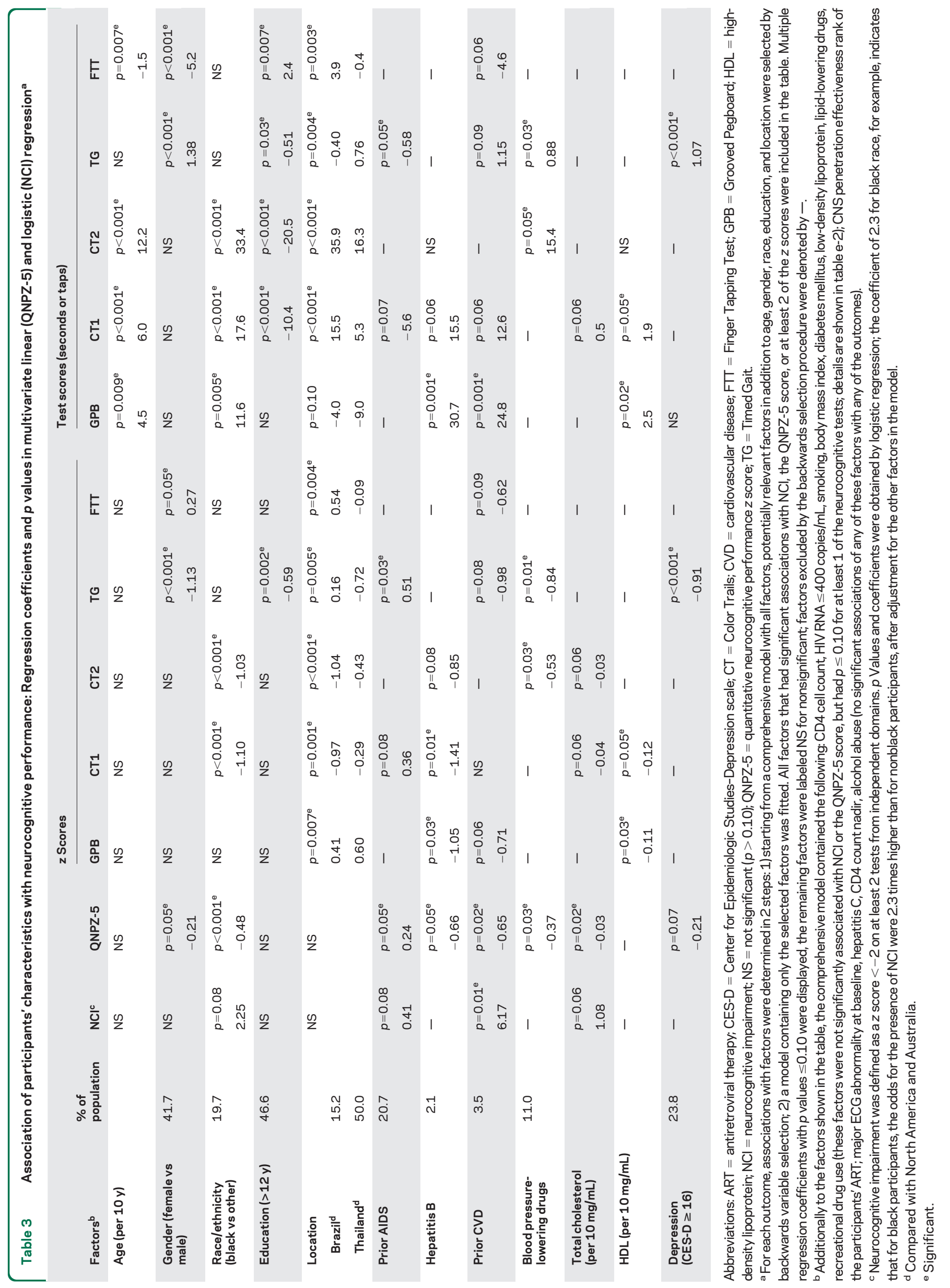


tive performance and the reason for this is unclear. The median CD 4 cell count was high $\left(536\right.$ cells $/ \mathrm{mm}^{3}$ ) but there was also no association of NCI with nadir CD4 counts. There was no evidence for an association of diabetes with impairment or poorer neurocognitive performance, although the lack of evidence may have been due to the low number of participants with diabetes. There was also no evidence for an association of the ART CNS penetration effectiveness rank with any of the neurocognitive performance scores. ART regimens with CNS penetration effectiveness ranks $\geq 2$ have been associated with improved neurocognitive performance ${ }^{26}$; in our study, $25 \%$ of participants had CNS penetration effectiveness ranks $\geq 2$. Contrary to expectations, participants with prior AIDS had a slightly increased estimated mean QNPZ-5 score (by 0.24, $p=$ 0.05 , table 3). This may be a statistical artifact due to confounding; in univariate analysis, those with and without prior AIDS had similar QNPZ-5 scores ( $p=$ 0.60 for difference), but those with prior AIDS had more CVD risk factors, including a higher body mass index (mean 25 vs 23) and more use of BP-lowering drugs (18\% vs $9 \%$ ).

Mean $z$ scores differed significantly between locations for each of the 5 neurocognitive tests; moreover, differences between locations persisted after adjusting for differences in age, sex, race, and education and other factors (table 3). At first glance, this leads to the hypothesis that the standard US norms were not appropriate for Brazil or Thailand. Within countries, however, $z$ scores also varied considerably between clinical sites (table 2, hierarchical model). Further adjustment for age, race, sex, and education decreased between-site variability in raw test scores, but had only minimal effect on the between-site variability in $z$ scores (data not shown). The latter suggests that our US norm $z$ scores reasonably standardized the test scores for these 4 demographic factors, and that the remaining variability between countries may be largely due to other unmeasured factors such as nutritional status, urban vs rural residency, cultural/ethnic factors, and test administration. It may be that if we had been able to more accurately measure and adjust for the education of participants, for example by assessing their reading level $^{39}$ instead of using years of education, we may have seen less between-country variability.

Twenty-four percent of participants met the study definition of depression; this proportion is commensurate with some studies but lower than others. ${ }^{15}$ Antidepressant use was not assessed.

One should interpret our study definition of NCI ( $z$ scores $<-2$ in 2 or more domains) with caution because 1) NCI was not confirmed clinically; 2) we used US norms in Brazilian and Thai populations; and 3) we assessed 4 cognitive domains only. ${ }^{19}$ Moreover, NCI may not always represent HAD or other $\mathrm{HIV}$-associated neurocognitive disorders because there is an overlap between the neurocognitive impairment associated with cerebrovascular disease $\mathrm{e}^{40}$ and HIV-associated CNS disease.

Study limitations include a moderate sample size, which limits the power to detect associations; a modest test battery; study-trained staff rather than neuropsychologists administering the neurocognitive tests; and the lack of local reference norms in Brazil and Thailand. Also, hypertension was not assessed directly, but extrapolated from the use of BP-lowering drugs. Finally, we evaluated multiple outcomes; some of the observed associations might be falsepositives, in particular where statistical significance was borderline.

Our findings suggest that, in HIV-infected persons with high CD4 cell counts, cardiovascularrelated insults may be more detrimental to neurocognitive functioning than factors more directly related to HIV. Given the high prevalence of cardiovascular risk factors in HIV-infected populations, this finding is important and warrants further investigation.

\section{AUTHOR CONTRIBUTIONS}

Statistical analysis was conducted by Dr. Grund and M. Roediger.

\section{ACKNOWLEDGMENT}

The authors thank the participants, site investigators, and staff for their contributions. Details of the clinical site investigators for the SMART Neurology substudy by site and country are presented in appendix e-2.

\section{DISCLOSURE}

Dr. Wright has served on a scientific advisory board for GlaxoSmithKline and receives research support from Gilead Sciences, Inc., Boehringer Ingelheim, Abbott, the National Health and Medical Research Council of Australia, and the NIH (NIMH/NINDS 1U01-AI068641 [Study Chair]). Dr. Grund has received research support from the NIH/NIAID (U01-AI068641 [Statistician], U01-AI042170 [Statistician], and U01AI46362 [Statistician]). Dr. Robertson has received speaker honoraria from GlaxoSmithKline, Abbott, Boehringer Ingelheim, and Clinical Care Options, and receives research support from the NIH (NIAID, supplement to 1U01AI068636-01 [PI], NIMH MH067751 [coinvestigator], NIAID U01-AI068641 [Co-PI], and NINDS R21NS0692 [coinvestigator]). Prof. Brew serves on scientific advisory boards for GlaxoSmithKline, ViiV Healthcare, Biogen Idec, and Merck Serono; has received funding for travel from Abbott; serves on the editorial boards of Open Virology, the International Journal for Tryptophan Research, Faculty of 1000 Medicine, the Journal of Neurovirology, and Neurobehavioral HIV Medicine; receives royalties from the publication of HIV Neurology (Oxford University Press, 2001) and Palliative Neurology (Cambridge University Press, 2006); has received speaker honoraria from GlaxoSmithKline, ViiV Healthcare, Boehringer Ingelheim, Abbott, and Biogen Idec; and receives/has received research support from Eli Lilly and Company, GlaxoSmithKline, ViiV Healthcare, Merck Serono, the National Health and Medical Research Council (NHMRC) of Australia, the NIH (R01 NS43103, co-PI), the University of New South Wales, and from St. Vincent's Clinic Research Foundation. Ms. Roediger has received research support from the NIH (NIAID U01-AI068641 [Statistician], NIAID U01-AI042170 [Statistician], and NIAID U01-AI46362 [Statistician]). Ms. Bain has received speaker honoraria from GlaxoSmithKline. Dr. Drummond and Dr. 
Vjecha report no disclosures. Prof. Hoy serves on scientific advisory boards for Gilead Sciences, Inc., Tibotec Therapeutics (Janssen), Merck Sharp \& Dohme, and Bristol-Myers Squibb; has received speaker honoraria from Gilead Sciences, Inc.; and receives research support from the National Health and Medical Research Council of Australia, Gilead Sciences, Inc., Merck Sharp \& Dohme, Tibotec Therapeutics, Bristol-Myers Squibb, and Boehringer Ingelheim. Ms. Miller and Dr. Penalva de Oliveira report no disclosures. Dr. Pumpradit has received funding for travel from Pfizer Inc. and has received research support from the Ministry of Public Health, Thailand. Dr. Shlay reports no disclosures. Dr. El-Sadr receives research support from the NIH/NIAID (5 U01 AI69466-04 [PI], 5 U01 AI69466-03S1 [PI], and R01AI083038-01 [PI]). Dr. Price has received speaker honoraria from the International AIDS Society and receives research support from Merck and Co, and from the NIH (NIDA P01DA026134 [Project PI], NIMH R01-MH62701 [PI], NIMH R21MH083520 [PI],. NIMH U01-MH083545 [Consultant], NIMH R01MH081772 [coinvestigator], NIMH/NIAIDU01-AI068641 [CoChair Neurology substudy Protocol], and NIMH/NIAID U01-AI38858 [Co-Chair AACTG study]).

Received January 30, 2010. Accepted in final form May 12, 2010

\section{REFERENCES}

1. McArthur JC, Hoover DR, Bacellar H, et al. Dementia in AIDS patients: incidence and risk factors: Multicenter AIDS Cohort Study. Neurology 1993;43:2245-2252.

2. Bhaskaran K, Mussini C, Antinori A, et al. Changes in the incidence and predictors of human immunodeficiency virus-associated dementia in the era of highly active antiretroviral therapy. Ann Neurol 2008;63:213-221.

3. El-Sadr WM, Lundgren JD, Neaton JD, et al. CD4+ count-guided interruption of antiretroviral treatment. N Engl J Med 2006;355:2283-2296.

4. Anstey KJ, von Sanden C, Salim A, O'Kearney R. Smoking as a risk factor for dementia and cognitive decline: a meta-analysis of prospective studies. Am J Epidemiol 2007;166:367-378.

5. Hayden KM, Zandi PP, Lyketsos CG, et al. Vascular risk factors for incident Alzheimer disease and vascular dementia: the Cache County study. Alzheimer Dis Assoc Disord 2006;20:93-100.

6. Anstey KJ, Lipnicki DM, Low LF. Cholesterol as a risk factor for dementia and cognitive decline: a systematic review of prospective studies with meta-analysis. Am J Geriatr Psychiatry 2008;16:343-354.

7. Silbert BS, Scott DA, Evered LA, Lewis MS, Maruff PT. Preexisting cognitive impairment in patients scheduled for elective coronary artery bypass graft surgery. Anesth Analg 2007;104:1023-1028.

8. Newman MF, Kirchner JL, Phillips-Bute B, et al. Longitudinal assessment of neurocognitive function after coronaryartery bypass surgery. N Engl J Med 2001;344:395-402.

9. Valcour VG, Shikuma CM, Shiramizu BT, et al. Diabetes, insulin resistance, and dementia among HIV-1-infected patients. J Acquir Immune Defic Syndr 2005;38:31-36.

10. Becker JT, Kingsley L, Mullen J, et al. Vascular risk factors, HIV serostatus, and cognitive dysfunction in gay and bisexual men. Neurology 2009;73:1292-1299.

11. Klove H. Clinical neuropsychology. Med Clin North Am 1963;47:1647-1658.

12. D'Elia LF, Satz P, Uchiyama CL, White T. Color Trails Test: Professional Manual. Odessa, FL: Psychological Assessment Resources; 1996.
13. Robertson KR, Parsons TD, Sidtis JJ, et al. Timed Gait test: normative data for the assessment of the AIDS dementia complex. J Clin Exp Neuropsychol 2006;28:10531064.

14. Reitan RM. Manual for Administration of Neuropsychological Test Batteries for Adults and Children. Indianapolis: Reitan RM; 1969.

15. Wright E, Brew B, Arayawichanont A, et al. Neurologic disorders are prevalent in HIV-positive outpatients in the Asia-Pacific region. Neurology 2008;71:50-56.

16. Price RW, Yiannoutsos CT, Clifford DB, et al. Neurological outcomes in late HIV infection: adverse impact of neurological impairment on survival and protective effect of antiviral therapy. AIDS 1999;13:1677-1685.

17. PAR Psychological Assessment Resources, Inc. PAI ${ }^{\circledR}$ Software Portfolio, 2006. Available at: www3.parinc.com. Accessed March 30, 2009.

18. Heaton RK, Miller SW, Taylor JJ, Grant I. Revised comprehensive norms for an expanded Halstead-Reitan battery: demographically adjusted neuropsychological norms for African Americans and Caucasian adults. Lutz, FL: PAR Psychological Assessment Resources, Inc.; 2004.

19. Antinori A, Arendt G, Becker JT, et al. Updated research nosology for HIV-associated neurocognitive disorders. Neurology 2007;69:1789-1799.

20. Radloff L. The CES-D Scale: a self-report depression scale for research in the general population. Appl Psychol Meas 1977;1:385-401.

21. Van Voorhees BW, Fogel J, Houston TK, Cooper LA, Wang NY, Ford DE. Beliefs and attitudes associated with the intention to not accept the diagnosis of depression among young adults. Ann Fam Med 2005;3:38-46.

22. Perczek R, Carver CS, Price AA, Pozo-Kaderman C. Coping, mood, and aspects of personality in Spanish translation and evidence of convergence with English versions. J Pers Assess 2000;74:63-87.

23. Fleck MP, Lima AF, Louzada S, et al. [Association of depressive symptoms and social functioning in primary care service, Brazil.] Rev Saude Publica 2002;36:431-438.

24. Center for Epidemiologic Studies-Depression Scale (CESD). Available at: http://www.dmh.go.th/test/cesd/cesd/. Accessed July 30, 2010.

25. Anderson KM, Odell PM, Wilson PW, Kannel WB. Cardiovascular disease risk profiles. Am Heart J 1991;121: 293-298.

26. Letendre S, Marquie-Beck J, Capparelli E, et al. Validation of the CNS Penetration-Effectiveness rank for quantifying antiretroviral penetration into the central nervous system. Arch Neurol 2008;65:65-70.

27. Samaras K, Wand H, Law M, Emery S, Cooper D, Carr A. Prevalence of metabolic syndrome in HIV-infected patients receiving highly active antiretroviral therapy using International Diabetes Foundation and Adult Treatment Panel III criteria: associations with insulin resistance, disturbed body fat compartmentalization, elevated C-reactive protein, and [corrected] hypoadiponectinemia. Diabetes Care 2007;30:113-119.

28. Brown TT, Li X, Cole SR, et al. Cumulative exposure to nucleoside analogue reverse transcriptase inhibitors is associated with insulin resistance markers in the Multicenter AIDS Cohort Study. AIDS 2005;19:1375-1383.

29. SMART/INSIGHT and D:A:D Study Groups. Use of nucleoside reverse transcriptase inhibitors and risk of myocar- 
dial infarction in HIV-infected patients. AIDS 2008;22: F17-F24.

30. Kivipelto M, Helkala EL, Laakso MP, et al. Midlife vascular risk factors and Alzheimer's disease in later life: longitudinal, population based study. BMJ 2001;322:1447-1451.

31. Craft S. The role of metabolic disorders in Alzheimer disease and vascular dementia: two roads converged. Arch Neurol 2009;66:300-305.

32. Engelhart MJ, Geerlings MI, Meijer J, et al. Inflammatory proteins in plasma and the risk of dementia: The Rotterdam Study. Arch Neurol 2004;61:668-672.

33. Sattar N, Murray HM, Welsh P, et al. Are markers of inflammation more strongly associated with risk for fatal than for nonfatal vascular events? PLoS Med 2009;6: e1000099.

34. Kowalska I, Straczkowski M, Nikolajuk A, et al. Insulin resistance, serum adiponectin, and proinflammatory markers in young subjects with the metabolic syndrome. Metabolism 2008;57:1539-1544.
35. Kuller LH, Tracy R, Belloso W, et al. Inflammatory and coagulation biomarkers and mortality in patients with HIV infection. PLoS Med 2008;5:e203.

36. Deeks SG. Immune dysfunction, inflammation, and accelerated aging in patients on antiretroviral therapy. Top HIV Med 2009; 17:118-123.

37. Guaraldi G, Zona S, Alexopoulos N, et al. Coronary aging in HIV-infected patients. Clin Infect Dis 2009;49:17561762.

38. Tisch S, Brew B. Parkinsonism in HIV-infected patients on highly active antiretroviral therapy. Neurology 2009; 73:401-403.

39. Manly JJ, Echemendia RJ. Race-specific norms: using the model of hypertension to understand issues of race, culture, and education in neuropsychology. Arch Clin Neuropsychol 2007;22:319-325.

40. Roman GC, Erkinjuntti T, Wallin A, Pantoni L, Chui HC. Subcortical ischaemic vascular dementia. Lancet Neurol 2002;1:426-436.

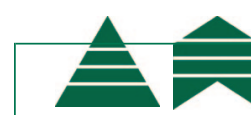

Editor's Note to Authors and Readers: Levels of Evidence coming to Neurology ${ }^{\circledR}$

Effective January 15, 2009, authors submitting Articles or Clinical/Scientific Notes to Neurology ${ }^{\circledR}$ that report on clinical therapeutic studies must state the study type, the primary research question(s), and the classification of level of evidence assigned to each question based on the classification scheme requirements shown below (left). While the authors will initially assign a level of evidence, the final level will be adjudicated by an independent team prior to publication. Ultimately, these levels can be translated into classes of recommendations for clinical care, as shown below (right). For more information, please access the articles and the editorial on the use of classification of levels of evidence published in Neurology. ${ }^{1-3}$

\section{REFERENCES}

1. French J, Gronseth G. Lost in a jungle of evidence: we need a compass. Neurology 2008;71:1634-1638.

2. Gronseth G, French J. Practice parameters and technology assessments: what they are, what they are not, and why you should care. Neurology 2008;71:1639-1643

3. Gross RA, Johnston KC. Levels of evidence: taking Neurology ${ }^{\circledR}$ to the next level. Neurology 2009;72:8-10.

\section{Classification scheme requirements for therapeutic questions}

Class I. A randomized, controlled clinical trial of the intervention of interest with masked or objective outcome assessment, in a representative population. Relevant baseline characteristics are presented and substantially equivalent among treatment groups or there is appropriate statistical adjustment for differences.

Class II. A randomized, controlled clinical trial of the intervention of interest in a representative population with masked or objective outcome assessment that lacks one criterion a-e in Class I or a prospective matched cohort study with masked or objective outcome assessment in a representative population that meets b-e in Class I. Relevant baseline characteristics are presented and substantially equivalent among treatment groups or there is appropriate statistical adjustment for differences.

Class III. All other controlled trials (including well-defined natural history controls or patients serving as their own controls) in a representative population, where outcome is independently assessed, or independently derived by objective outcome measurements.

Class IV. Studies not meeting Class I, II, or III criteria including consensus or expert opinion.
AAN classification of recommendations

$A=$ Established as effective, ineffective, or harmful (or established as useful/predictive or not useful/predictive) for the given condition in the specified population. (Level $\mathrm{A}$ rating requires at least two consistent Class I studies.)

$B=$ Probably effective, ineffective, or harmful (or probably useful/predictive or not useful/predictive) for the given condition in the specified population. (Level B rating requires at least one Class I study or two consistent Class II studies.)

\section{7 . Doscihluaffactiva inaffectiva or harmf}

$\mathrm{C}=$ Possibly effective, ineffective, or harmful (or possibly useful/predictive or not useful/predictive) for the given condition in the specified population. (Level $\mathrm{C}$ rating requires at least one Class II study or two consistent Class III studies.)

$\mathrm{U}=$ Data inadequate or conflicting; given current knowledge, treatment (test, predictor) is unproven.

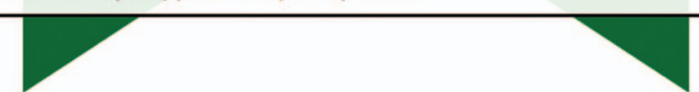

